\title{
Erratum to: Increased phosphatidylcholine (16:0/16:0) in the folliculus lymphaticus of Warthin tumor
}

\author{
Qian He • Yoshinori Takizawa • Takahiro Hayasaka • \\ Noritaka Masaki • Yukiko Kusama • Jiping Su • \\ Hiroyuki Mineta $\cdot$ Mitsutoshi Setou
}

Received: 13 August 2014 / Accepted: 13 August 2014 / Published online: 5 September 2014

(C) Springer-Verlag Berlin Heidelberg 2014

\section{Erratum to: Anal Bioanal Chem \\ DOI 10.1007/s00216-014-7890-9}

The authors would like to call your attention to the following:

The legend of Figure 6 should read "Location of PC (16:0/ 16:0) in the War-T region. The ion images of $\mathrm{m} / \mathrm{z} 772.5$, identified as $[\mathrm{PC}(16: 0 / 16: 0)+\mathrm{K}]^{+}$, were merged with the HE staining image for case 1 . According to the HE staining, we discriminated the neoplastic epithelium and lymphoid stroma on the basis of the description in Fig. 1. The ion image showed that the signal at $[\mathrm{PC}(16: 0 / 16: 0)+\mathrm{K}]^{+}(\mathrm{m} / \mathrm{z} 772.5)$ was more intense in the lymphoid stroma and performed clusters in the regions circled by red lines, folliculus lymphaticus".

The sentence "In our study, most of the lipids detected in the War-T regions included two double bonds, such as PC (16:0/18:2)" should read: "In our study, most of the lipids

Published in the topical collection New Applications of Mass Spectrometry in Biomedicine with guest editors Kazuo Igarashi, Mitsutoshi Setou, and Toshimitsu Niwa.

The online version of the original article can be found at http://dx.doi.org/ 10.1007/s00216-014-7890-9.

\section{Q. He J. Su $(\bowtie)$}

Department of Otolaryngology-Head and Neck Surgery,

First Affiliated Hospital of Guangxi Medical University,

No. 6 Shuangyong Rd, Nanning 530021, China

e-mail:ymsu2@126.com

Q. He $\cdot$ Y. Takizawa $\cdot$ H. Mineta

Department of Otorhinolaryngology/Head \& Neck Surgery,

Hamamatsu University School of Medicine, 1-20-1 Handayama,

Higashi-ku, Hamamatsu, Shizuoka 431-3192, Japan detected in the War-T regions included more than two double bonds, such as PC (16:0/20:3)".

The paragraph under Figure 6 should read: "However the signal at $[\mathrm{PC}(16: 0 / 16: 0)+\mathrm{K}]^{+}(\mathrm{m} / z$ 772.5) showed a different localization compared to the other War-T signals. The merged ion images of $[\mathrm{PC}(16: 0 / 16: 0)+\mathrm{K}]^{+}(\mathrm{m} / \mathrm{z} 772.5)$ and $[\mathrm{PC}(36: 2)+\mathrm{K}]^{+}(\mathrm{m} / \mathrm{z}$ 824.5) revealed a clear positional discrimination between these two biomolecules. By comparison with the HE staining images, the signal at $[\mathrm{PC}(36: 2)+\mathrm{K}]^{+}(\mathrm{m} / \mathrm{z}$ 824.5) was found at neoplastic epithelium. In contrast, the signal at $[\mathrm{PC}(16: 0 / 16: 0)+\mathrm{K}]^{+}(\mathrm{m} / \mathrm{z} 772.5)$ was located mainly in lymphoid stroma (Fig. 4). Especially, the folliculus lymphaticus in the War-T region exhibited high signal intensities at $[\mathrm{PC}(16: 0 / 16: 0)+\mathrm{K}]^{+}(\mathrm{m} / \mathrm{z} 772.5) "$

Unfortunately, there was a mistake in Figure 5 of this contribution. Please find the correct Figure 5 below:

Q. He $\cdot$ T. Hayasaka $\cdot$ N. Masaki $\cdot$ M. Setou $(\square)$

Department of Cell Biology and Anatomy, Hamamatsu University School of Medicine, 1-20-1 Handayama, Higashi-ku, Hamamatsu, Shizuoka 431-3192, Japan

e-mail: setou@hama-med.ac.jp

Y. Kusama

Department of Diagnostic Pathology, Hamamatsu University School of Medicine, 1-20-1 Handayama, Higashi-ku, Hamamatsu,

Shizuoka 431-3192, Japan 

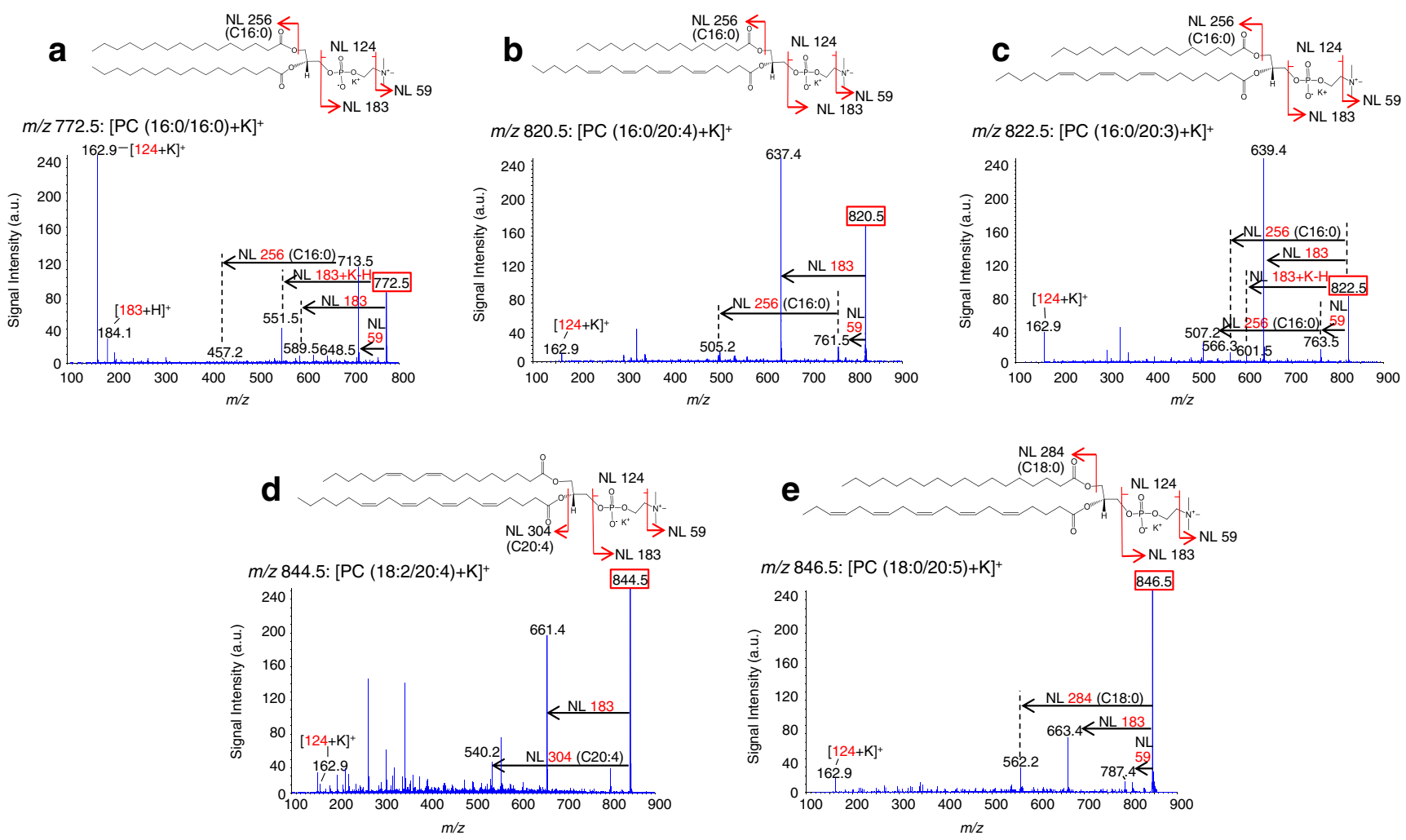

Fig. 5 Molecular identification by the MS/MS analysis of tissue sections. The precursor ions were $\mathrm{m} / \mathrm{z} 772.5$ (a), m/z 820.5 (b), m/z 822.5 (c), m/z 844.5 (d) and $\mathrm{m} / \mathrm{z} 846.5$ (e). From the product ion spectrum, a molecule the same manner, the signals at $\mathrm{m} / \mathrm{z} 820.5, \mathrm{~m} / \mathrm{z} 822.5, \mathrm{~m} / \mathrm{z} 844.5$ and $\mathrm{m} / \mathrm{z}$ 846.5 were identified as [PC $(16: 0 / 20: 4)+\mathrm{K}]+$, [PC $(16: 0 / 20: 3)+\mathrm{K}]+$, $[\mathrm{PC}(18: 2 / 20: 4)+\mathrm{K}]+$ and $[\mathrm{PC}(18: 0 / 20: 5)+\mathrm{K}]+$, respectively corresponding to $\mathrm{m} / \mathrm{z} 772.5$ was identified as [PC $(16: 0 / 16: 0)+\mathrm{K}]+$. In 